\title{
Magnetic Resonance Imaging (MRI) Phenotypes May Provide Additional Information for Risk Stratification for Encapsulated Papillary Carcinoma of the Breast
}

This article was published in the following Dove Press journal: Cancer Management and Research

Wen-jie Tang, ${ }^{1, *}$ YunShi Liang, $\mathbb{D}^{2, *}$ Jing Yan, $\mathbb{D}^{3}$ Yue Hu, ${ }^{4}$ Mei-Li Sun, ${ }^{5}$ GuoShun Liu,' Xiao-Huan Pan, ${ }^{6}$ Xin-Hua Wei,' XinQing Jiang,' Yuan Guo'

'Department of Radiology, Guangzhou First People's Hospital, School of Medicine, South China University of Technology, Guangzhou 510180, People's Republic of China; '2Department of Pathology, Guangzhou First People's Hospital, School of Medicine, South China University of Technology, Guangzhou 510180, People's Republic of China; ${ }^{3}$ Department of MRI, The First Affiliated Hospital of Zhengzhou University, Zhengzhou 450052, People's Republic of China; ${ }^{4}$ Department of Ultrasound, Sun Yat-Sen Memorial Hospital Sun Yat-Sen University, Guangzhou 510120 , People's Republic of China; ${ }^{5}$ Department of Radiology, Sun Yat-Sen University Cancer Center, Guangzhou 5 10060, People's Republic of China; ${ }^{6}$ Department of Radiology, The First Affiliated Hospital of Guangzhou Medical University, Guangzhou 5 I0120, People's Republic of China

*These authors contributed equally to this work

Correspondence: Yuan Guo; Xin-Qing Jiang

Department of Radiology, Guangzhou First People's Hospital, School of Medicine, South China University of Technology, No. I Panfu Road,

Guangzhou, Guangdong 510180, People's Republic of China

Tel +86 I36-3249-8569;

$+86|8|-028 \mid-2838$

Fax +86-20-81048873

Email eyguoyuan@scut.edu.cn;

eyjiangxq@scut.edu.cn
Background: Encapsulated papillary carcinoma (EPC) of the breast is a rare entity. EPC can be underappreciated on percutaneous biopsy, which may require additional procedures if invasion is not recognized preoperatively. We aimed to investigate the magnetic resonance imaging (MRI) phenotypes correlated with preoperative pathological risk stratification for clinical guidance.

Materials and Methods: The preoperative MRI scans of 30 patients diagnosed with 36 EPCs in multiple centers between August 2015 and February 2020 were reviewed by two breast radiologists. According to the WHO classification published in 2019, EPCs were classified into two pathological subtypes: encapsulated papillary carcinoma and encapsulated papillary carcinoma with invasion. Clinicopathological analysis of the two subtypes and MR feature analysis were performed.

Results: Evaluation of the MRI phenotypes and pathological subtype information revealed that not circumscribed $(\mathrm{P}=0.04)$ was more common in EPCs with invasion than in EPCs. There was a significant difference in the age of patients $(\mathrm{P}=0.05)$, and the risk increased with age. The maximum diameter of the tumor increased with tumor risk, but there was no significant difference $(\mathrm{P}=0.36)$. Nearly half of the EPC with invasion patients showed hyperintensity on T1WI $(\mathrm{P}=0.19)$. A total of $63.6 \%$ of the EPC with invasion group showed non-mass enhancement surrounding $(\mathrm{P}=0.85)$. In addition, 29 patients $(96.7 \%)$ had no axillary lymph node metastasis, and only one patient with EPC with invasion had axillary lymph node metastasis. Further pathological information analysis of EPCs showed that higher Ki-67 levels were more common in patients with EPCs with invasion $(\mathrm{P}=0.04)$. A total of 29 patients $(96.7 \%$ ) had the luminal phenotype, and one patient with EPC with invasion had the Her-2-positive phenotype.

Conclusion: The margin, age and Ki-67 level were the key features for EPC risk stratification. In addition, these MRI signs, including a larger tumor, non-mass enhancement surrounding and axillary lymph node metastasis, may be suggestive of a high-risk stratification. Therefore, MRI phenotypes may provide additional information for the risk stratification of EPCs.

Keywords: carcinoma, papillary, breast neoplasms, magnetic resonance imaging, pathology

\section{Introduction}

Encapsulated papillary carcinoma (EPC) of the breast, also commonly referred to as intracystic papillary carcinoma or encysted papillary carcinoma, is a rare breast cancer subtype, accounting for $0.5 \%$ to $2.0 \%$ of all breast tumors. ${ }^{1,2}$ EPC has been 
considered a variant of ductal carcinoma in the past. The latest World Health Organization (WHO) Working Group's classification of breast tumors defines EPC as a separate subtype of papillary lesions that are characterized by a fibrous capsule surrounding the tumor, typically without a layer of myoepithelial cells. ${ }^{2,3}$

According to the latest edition of the WHO Classification of Tumors of the Breast published in 2019, ${ }^{4}$ EPC is classified into encapsulated papillary carcinoma and encapsulated papillary carcinoma with invasion. Several studies have demonstrated that the classification has clear prognostic significance, suggesting that the prognosis of EPC is excellent compared with that of EPC with invasion. ${ }^{5-7}$ Moreover, the clinical management of these two types is also different. Local excision with negative surgical margins seems to be sufficient for pure EPC; however, EPC with invasion are staged and managed according to characteristics of the invasive component. ${ }^{6}$ Therefore, a preoperative assessment of different risk stratifications of EPC is of great significance for treatment decision-making and prognosis prediction.

Percutaneous biopsy is a good method for preoperative diagnosis and is widely used in the preoperative diagnosis of breast lesions. However, the diagnosis of EPC with invasion with both core needle biopsy (CNB) and vacuum-assisted biopsy (VAB) may be challenging. ${ }^{1,8}$ Because of the limited size of the biopsy, the invasive nature of the lesion remains difficult to establish. ${ }^{9}$ Therefore, it is particularly important to use imaging methods for the noninvasive preoperative evaluation of EPC. Among mammography, ultrasound and magnetic resonance imaging (MRI), MRI is widely applied to obtain an accurate preoperative diagnosis of breast tumors because of its high resolution and multiple parameters. ${ }^{10,11}$ A few reports ${ }^{12-14}$ have discussed the morphological characteristics of EPC based on MRI, and their results described EPC as a complex cystic mass containing solid components, and contrast-enhanced images showed enhancement of the cystic wall and mural nodules. However, the number of patients in the previous studies was relatively small, and no research reported the correlation between risk stratification and MR features. Therefore, the present study aimed to investigate MRI phenotypes correlated with preoperative pathological risk stratification.

\section{Materials and Methods}

\section{Patients}

The institutional Ethics Committee of the Guangzhou First People's Hospital, South China University of Technology approved this retrospective study. The requirement for informed consent was waived by the ethics committee due to the nature of the study, and no personal information was disclosed. This study strictly abides by the principles of the World Medical Association Declaration of Helsinki. Patients with pathologically confirmed EPC were included in the study. Patients who did not undergo surgery, did not have MR images acquired after biopsy or surgery or underwent MRI at an outside hospital were excluded from the study. A total of 2956 patients with breast cancer from August 2015 to February 2020 were recruited from four hospitals. Among them, the 51 patients who underwent surgery and definitive diagnoses were provided by histopathology. Finally, 30 patients had complete clinical data and underwent breast MRI before surgery.

\section{MRI Technique}

MRI was performed using a 1.5-T Dedicated spiral breast MRI system (Philips, Achieva Systems, Netherlands) $(\mathrm{n}=14)$; (United Imaging, uMR 560, China) $(\mathrm{n}=6)$ or 3.0-T Dedicated spiral breast MRI system (Siemens, Magnetom Skyra, Germany) ( $\mathrm{n}=10)$ with patients in the prone position. First, axial T1-weighted images and fatsuppressed T2-weighted images of the breasts were obtained. Second, transverse diffusion-weighted imaging (DWI) was performed using a single-shot spin-echo echo-planar imaging sequence with the following parameters: repetition time/echo time (TR/TE), 3800/78 ms; field of view, $350 \times 200 \mathrm{~mm}^{2}$; matrix, 156 $\times 156$; slice thickness, $4 \mathrm{~mm} ; 27$ slices; voxel size: $2.0 \times 2.0 \times 4.0 \mathrm{~mm}^{3}$; b value: 50 and $800 \mathrm{~s} / \mathrm{mm} 2$; number of averages, 1 ; acquisition time, $103 \mathrm{~s}$. Third, the gadoliniumbased agent gadopentetate dimeglumine (Gd-DTPA, Magnevist; Bayer Healthcare, Berlin, Germany) was intravenously injected at a dose of $0.2 \mathrm{~mL} / \mathrm{kg}$ body weight and a rate of $1.5 \mathrm{~mL} / \mathrm{s}$, followed by a $20-\mathrm{mL}$ saline flush performed with a high-pressure injector. Axial 3D fat-saturated T1-weighted images were obtained just before contrast administration and at 6 consecutive time points (in $60 \mathrm{~s}$ intervals) following the administration of the Gd-DTPA contrast agent, with the following parameters: TR/TE, 5.1/2.1 msec; flip angle, 10; field of view, $320 \times 320 \mathrm{~mm}^{2}$; matrix, $400 \times 70$; slice thickness, $2.4 \mathrm{~mm}$.

\section{Image Analysis}

The MR images were evaluated independently by two radiologists experienced in breast MRI (YG. and WT. with 13 and 7 years of experience, respectively). All features were determined by consensus. According to the latest Breast 
Imaging Reporting and Data System (BI-RADS) - MRI, 5th edition guidelines, ${ }^{15}$ the following descriptors were used in the analysis: age, location, number of masses, longest diameter, margin (circumscribed or not circumscribed includes irregular and spiculated), cystic or solid component ratio, T1weighted imaging (T1WI) signal intensity (higher or lower than normal breast tissue signal intensity), T2-weighted imaging (T2WI) signal intensity (heterogeneous or homogeneous), enhancement type, TIC pattern (progressive, plateau, wash-out), non-mass enhancement (absent or present), DWI signal, apparent diffusion coefficient (ADC) value and $\mathrm{ADC}$ ratio.

Depending on the cystic and solid components of EPCs, the tumors were divided into three types: mainly solid mass (80-100\% solid component), mainly cystic mass (80-100\% cystic component), and complex cystic and solid mass. In addition, we additionally evaluated the $\mathrm{ADC}$ ratio, which is calculated as the $\mathrm{ADC}$ value of necrosis or cystoid degeneration to the wall ADC value.

\section{Pathologic Analysis}

Patients were diagnosed with EPC according to the latest WHO Classification of Tumors of the Breast. All patients were divided into two groups: EPC $(n=19)$ and EPC with invasion ( $\mathrm{n}=11)$. The Ki-67 index was evaluated and classified into a negative group ( $\mathrm{Ki}-67<20 \%)$ and a positive group (Ki-67 $\geq 20 \%$ ).

\section{Statistical Analysis}

Continuous variables are expressed as the median and IQR and were compared with the unpaired $t$-test if the data were normally distributed or the Mann-Whitney $U$-test if the data were not normally distributed. A nonparametric test (Mann-Whitney) was used to test other nonparametric variables within each group. SPSS (version 19.0, SPSS, Chicago, USA) was used to perform statistical analyses. $P$ values $\leq 0.05$ were considered statistically significant.

\section{Results}

\section{Patient Characteristics}

In this study, 36 EPCs were identified in 30 patients (24-86 years, median 60 years), with a median diameter of $23.5 \mathrm{~mm}$. Eight women were premenopausal and 22 were postmenopausal. Among all patients, 23 patients had the first symptom of breast lumps, 3 patients showed nipple discharge and 4 patients experienced breast pain (10 cases were found by ultrasound screening examination, and 20 cases were found by diagnostic ultrasound examination). Twenty patients had a long history of symptoms (more than 1 year), and 12 of them had significantly increased symptoms recently. Four patients had more than one mass. One patient had a breast cancer history at the contralateral breast five years ago. The EPC was in the right breast in 17 cases and the left breast in 13 cases. Based on the WHO classification published in 2019, 19 cases were EPC and 11 cases were EPC with invasion. The median time for follow-up was 30.5 months (12-60 months). At the end of follow-up, 24 (80\%) patients were still alive, and $6(20 \%)$ patients were lost

Table I The Characteristics of 30 Patients with EPCs

\begin{tabular}{|c|c|c|}
\hline Character & Number & $\%$ \\
\hline Age-years (median, IQR) & $60(50-69)$ & \\
\hline Diameter-mm (median, IQR) & $23.5(19.3-34.0)$ & \\
\hline \multicolumn{3}{|l|}{ Menopause status } \\
\hline Pre-menopausal & 8 & 26.7 \\
\hline Post-menopausal & 22 & 73.3 \\
\hline \multicolumn{3}{|l|}{ Manifestation } \\
\hline Nipple discharge & 3 & 10.0 \\
\hline Breast lumps & 23 & 76.7 \\
\hline Pain & 4 & 13.3 \\
\hline \multicolumn{3}{|l|}{ Location } \\
\hline Left & 13 & 43.3 \\
\hline Right & 17 & 56.7 \\
\hline \multicolumn{3}{|l|}{ Cancer History } \\
\hline Breast cancer history & I & 3.3 \\
\hline No cancer history & 29 & 96.7 \\
\hline \multicolumn{3}{|l|}{ History } \\
\hline Less than I year & 10 & 33.3 \\
\hline More than I year & 20 & 66.7 \\
\hline Stable continuously & 8 & 26.7 \\
\hline Rapid growth recently & 12 & 40.0 \\
\hline \multicolumn{3}{|l|}{ Masses number } \\
\hline Single & 26 & 86.7 \\
\hline Multiple & 4 & 13.3 \\
\hline \multicolumn{3}{|l|}{ Pathology } \\
\hline EPC & 19 & 63.3 \\
\hline EPC with invasion & 11 & 36.7 \\
\hline Median time for follow-up (months) & 30.5 & \\
\hline \multicolumn{3}{|l|}{ Prognosis } \\
\hline Alive & 24 & 80.0 \\
\hline Lost follow-up & 6 & 20.0 \\
\hline Dead & 0 & 0.0 \\
\hline
\end{tabular}


to follow-up. One patient had invasive carcinoma of the contralateral breast two years later. The characteristics of all studied samples are summarized in Table 1.

\section{Imaging Characteristics}

Of the studied samples, 14 EPCs were round or oval masses with regular margins, while 16 masses were not circumscribed. On T1WI, EPCs showed various signals from hypointensity $(\mathrm{n}=22)$ to mixed hyperintensity $(\mathrm{n}=8)$. Tumor consistency was divided into three main groups: 12 cases (12/30) showed complex cystic and solid masses, 13 cases (13/30) showed mainly solid masses and 5 cases (5/ 30) showed mainly cystic masses. On the postcontrast images, three lesions displayed minor or mild enhancement, and 27 masses showed moderate heterogeneous enhancement. Time-signal intensity curve (TIC) pattern:

Table 2 Association Between EPC Imaging Findings and Pathological Features

\begin{tabular}{|c|c|c|c|c|}
\hline MR Character & $\operatorname{EPC}(n=19)$ & EPC with Invasion $(n=I I)$ & Statistical value & P value \\
\hline Age-years (median, IQR) & $57(47-67)$ & $66(58-74)$ & 2.06 & 0.05 \\
\hline Diameter-mm (median, IQR) & $21.0(19.5-29.0)$ & $24.0(19.5-47.0)$ & 0.93 & 0.36 \\
\hline Number (single/multiple) & $18 / 1$ & $8 / 3$ & -1.68 & 0.33 \\
\hline ADC value $\left(\times 10^{-6} \mathrm{~mm}^{2} / \mathrm{s}\right)$ & $843.4 \pm 103.2$ & $876.3 \pm 128.5$ & 0.77 & 0.45 \\
\hline ADC ratio: ADC (necrosis or cystoid degeneration)/ADC (wall) & $2.50 \pm 0.23$ & $2.4 I \pm 0.33$ & 0.98 & 0.34 \\
\hline TI hyperintensity & & & -1.74 & 0.19 \\
\hline Present & 3 & 5 & & \\
\hline Absent & 16 & 6 & & \\
\hline T2 signals & & & -0.75 & 0.61 \\
\hline Hypointensity-isointensity & 3 & 3 & & \\
\hline Mixed & 16 & 8 & & \\
\hline DWI signals & & & -0.38 & 0.80 \\
\hline Hyperintensity & 4 & 3 & & \\
\hline Mixed & 15 & 8 & & \\
\hline Enhancement (solid) & & & -1.12 & 0.58 \\
\hline Minor-mild & I & 2 & & \\
\hline Moderate and above & 18 & 9 & & \\
\hline TIC pattern & & & 0.09 & 0.77 \\
\hline Progressive & 2 & 1 & & \\
\hline Plateau & 14 & 9 & & \\
\hline Wash-out & 3 & 1 & & \\
\hline Non-mass enhancement surrounding & & & -0.23 & 0.85 \\
\hline Present & 7 & 7 & & \\
\hline Absent & 12 & 4 & & \\
\hline Margin & & & -2.34 & 0.04 \\
\hline Circumscribed & 12 & 2 & & \\
\hline Not Circumscribed & 7 & 9 & & \\
\hline Component & & & 1.83 & 0.18 \\
\hline Mainly solid mass (80-100\% solid component) & 9 & 4 & & \\
\hline Mainly cystic mass ( $80-100 \%$ cystic component) & 5 & 0 & & \\
\hline Complex cystic and solid mass (others) & 5 & 7 & & \\
\hline Dilated duct surrounding & & & -2.20 & 0.09 \\
\hline Present & 3 & 6 & & \\
\hline Absent & 16 & 5 & & \\
\hline Axillary Lymph node & & & -1.31 & 0.70 \\
\hline Positive & 0 & 1 & & \\
\hline Negative & 19 & 10 & & \\
\hline
\end{tabular}


23 cases (23/30) demonstrated plateau enhancement curves on dynamic contrast-enhanced MRI (DCE-MRI), while 3 cases (3/30) demonstrated progressive enhancement curves and 4 cases (4/30) demonstrated wash-out enhancement curves.

\section{Association Between EPC Imaging Findings and Pathological Features}

The comparison of multiparameter MR findings between the EPC and EPC with invasion groups is presented in Table 2, and the schematic diagram is shown in Figure 1. Based on the evaluation of MRI phenotype and pathological subtype information, not circumscribed $(\mathrm{P}=0.04)$ was more easily found in EPCs with invasion (Figure 2) compared with EPCs (Figure 3). In addition, the risk increased with age, and there was a significant difference $(\mathrm{P}=0.05)$.

The maximum diameter of the tumor increased with risk, while there was no significant difference $(\mathrm{P}=0.36)$. Nearly half of the EPC with invasion patients (5/11) showed hyperintensity on T1WI and surrounding dilated ducts, although there was no significant difference $(\mathrm{P}=0.19,0.09)$. A total of
$63.6 \%$ of the EPC with the invasion group showed non-mass enhancement surrounding, while only $36.8 \%$ of the EPC group showed the same characteristics $(\mathrm{P}=0.85)$. In addition, 29 patients $(96.7 \%)$ had no axillary lymph node metastasis, and only one EPC with invasion patient with axillary lymph node metastasis was found.

\section{Surgical Options in Different EPC Types}

In the EPC group $(n=19)$, breast-conserving surgery was performed in 14 cases, and simple mastectomy was performed in 5 cases. In the EPC with invasion group $(n=11)$, breast-conserving surgery was performed in four cases and simple mastectomy in seven cases. In this study, the concordance rates of $\mathrm{CNB}$ and VAB were $36.4 \%$ and $62.5 \%$, respectively. All details are shown in Table 3.

\section{The Risk Stratification for EPC of the Breast and Information of Pathology}

In our study, $10(10 / 19)$ EPC cases showed a Ki-67 $\geq 20 \%$, and $10(10 / 11)$ EPC with invasion cases showed a Ki$67 \geq 20 \%$. Higher Ki-67 levels were more common in
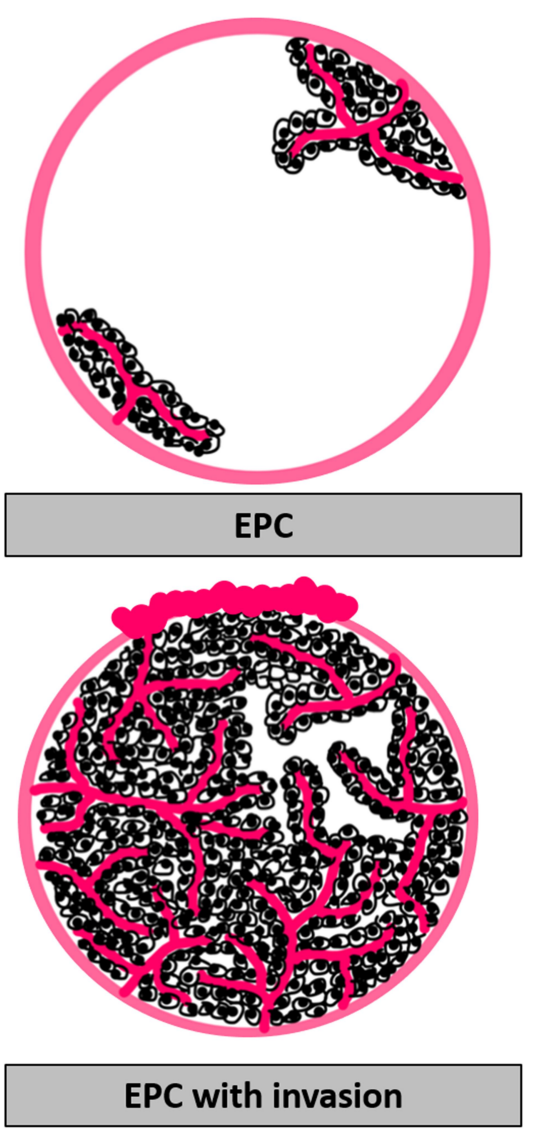

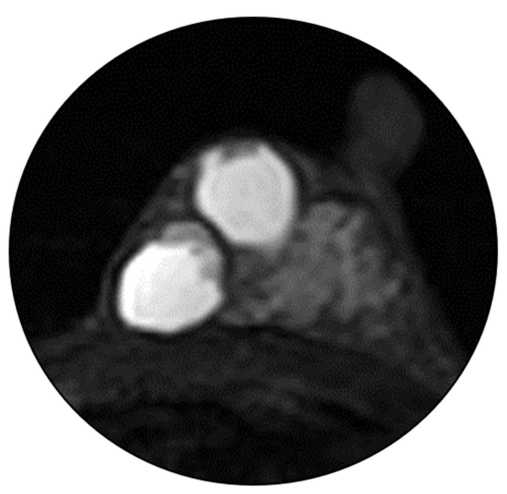

MRI (mainly cystic mass)

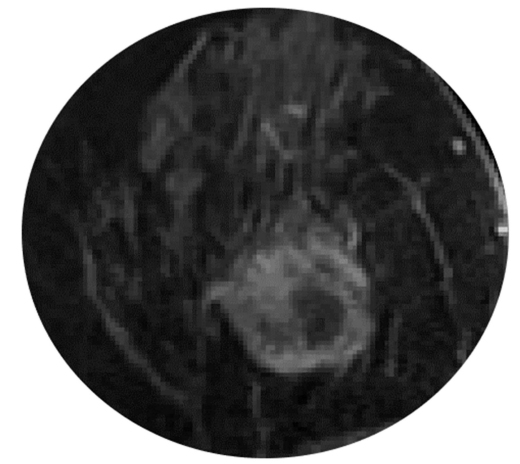

MRI (cystic and solid mass)

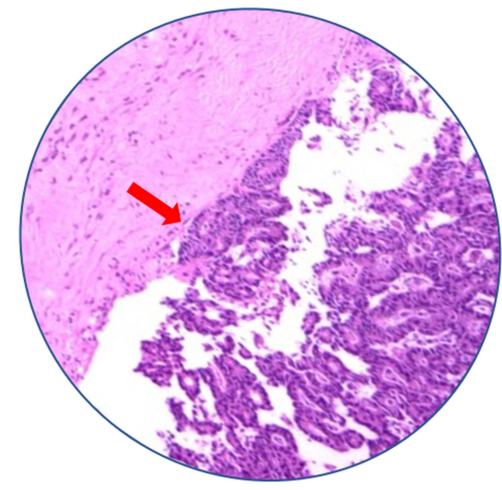

Pathology (without invasion)

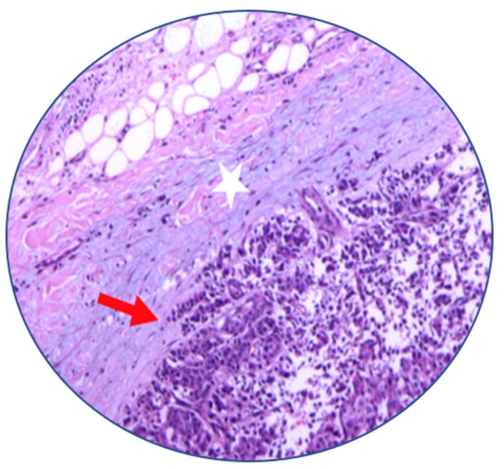

Pathology (with invasion)

Figure I According to the WHO classification published in 2019, the EPCs were classified into two pathological subtypes: EPC and EPC with invasion. The above is the schematic diagram, MRI and pathological diagram. 


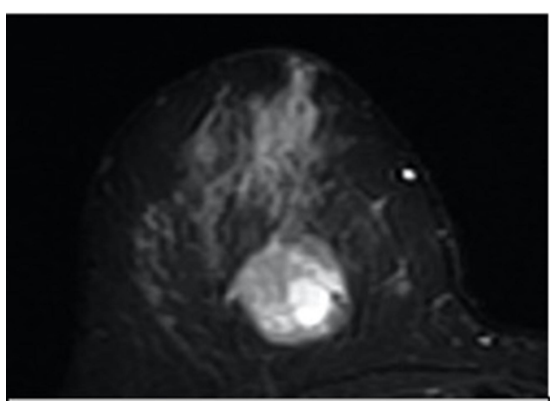

A. T2WI-fat suppression (TRA)

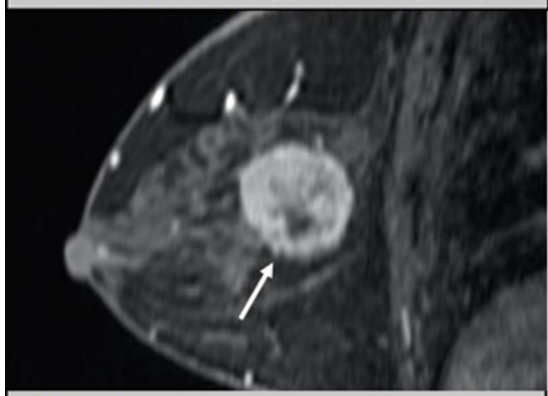

D. T1WI-fat suppression+C (SAG)

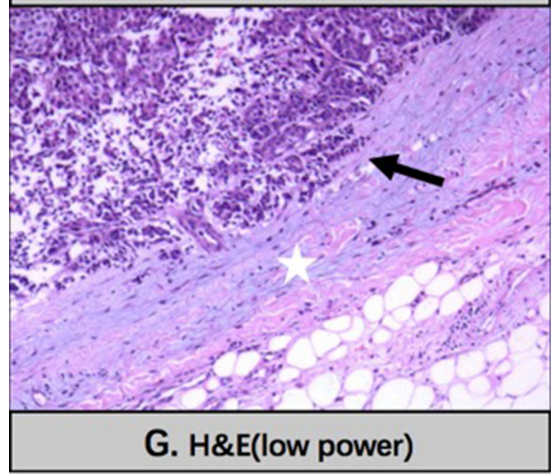

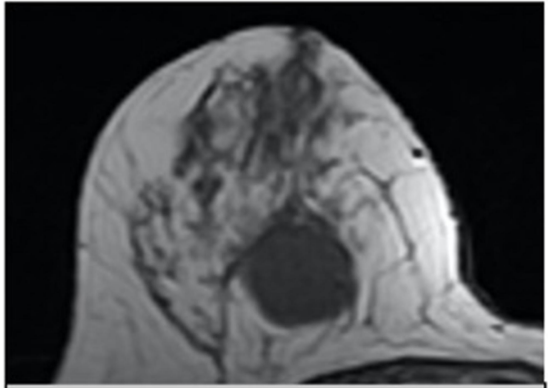

B. T1WI (TRA)

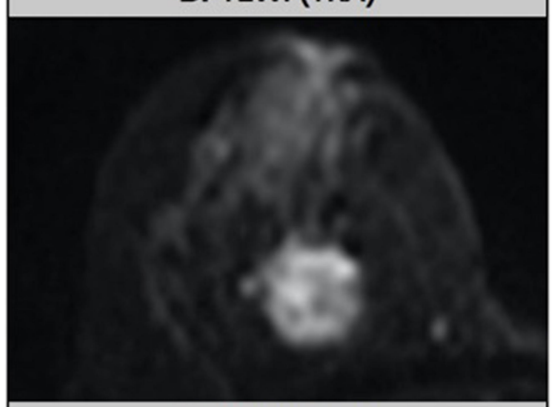

E. DWI $(b=800)$

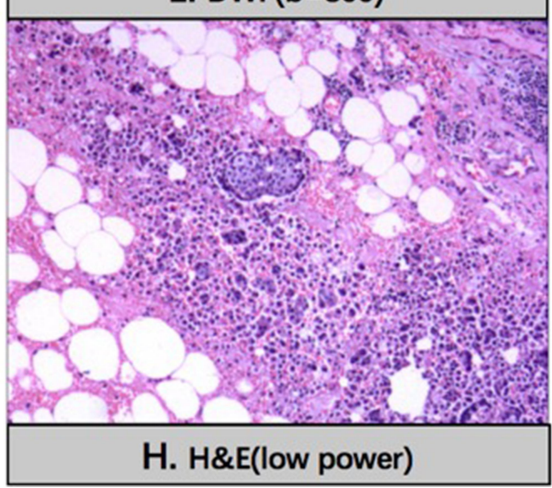

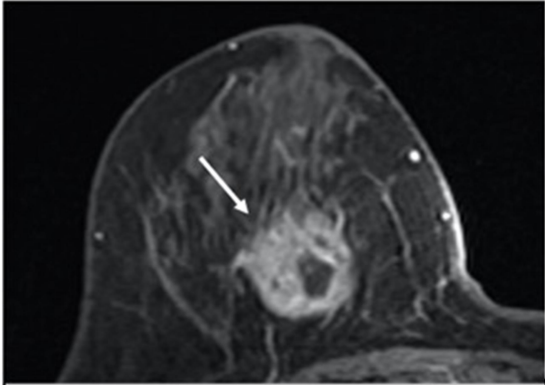

C. T1WI-fat suppression +C (TRA)

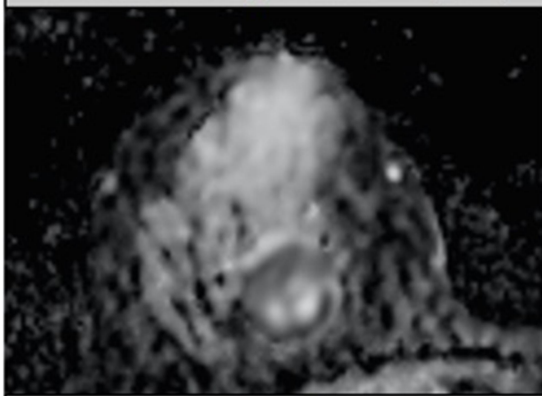

F. ADC

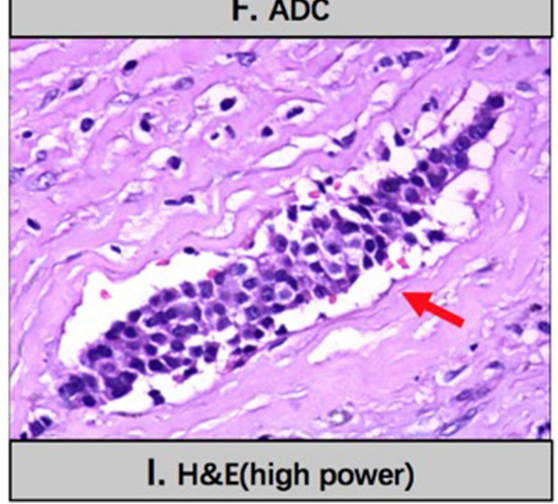

Figure 2 A 65-year-old woman with EPC with invasion in the right breast. Axial T2WI with fat suppression (A) showed a mass with mixed hyperintensity to hyperintensity. Axial TIWI (B) showed a mass with hypointensity. Axial fat-suppressed contrast-enhanced TIWI (C) and sagittal (D) showed indistinct tumor margin (white arrow). DWI (E) showed a high signal mass, and the signal of the ADC map (F) was complex. (G), Hematoxylin and eosin (H\&E) staining (low power) of the breast mass illustrates fibrous capsule was invaded by tumor cells (the pentagram showed fibrous capsule, the black arrow showed tumor cells). (H), H\&E staining (low power) showed tumor cell infiltration in the stroma surrounding the tumor, and vascular cancer embolus (red arrow) were seen at high power (I).

EPC patients with invasion $(\mathrm{P}=0.04)$. All EPC cases were the luminal phenotype (19/19), whereas among the EPC with invasion cases, $10(10 / 11)$ cases were the luminal phenotype, and $1(1 / 11)$ case was the Her-2-positive phenotype. All details are shown in Table 4.

\section{Discussion}

In our study, we investigated the value of MRI characteristics for the preoperative risk stratification of EPC. In general, not circumscribed of tumor margin and age of patients were two important predictors of pathological invasiveness. A larger tumor, non-mass enhancement surrounding and axillary lymph node metastasis may indicate a poor pathologic type. In addition, pathological information, including Ki-67 and molecular subtypes, further indicated the risk of these two pathological types.

In our study, the majority of EPC patients showed classic features of cystic-solid mixed masses with mural nodules, which was similar to those of previous studies. $^{13,14}$ However, we found a lack of studies using MRI findings to assess the risk stratification of the disease. To further investigate the relationship between MRI findings and the risk stratification of EPC, 30 EPC patients with different pathological risk stratifications were enrolled from multiple centers. In general, margins and age of patients played considerable roles in the prediction 


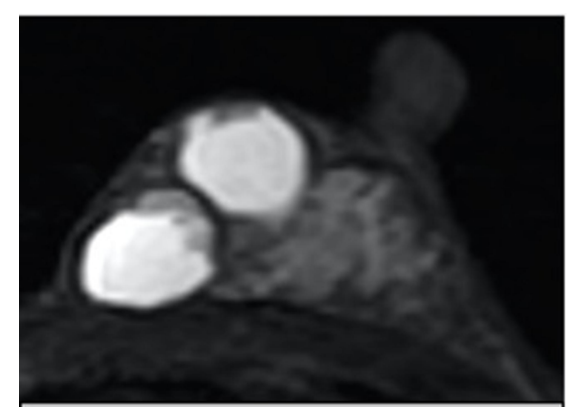

A. T2WI-fat suppression (TRA)

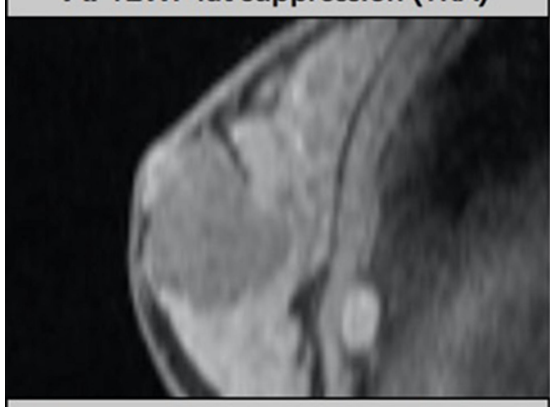

D. T1WI-fat suppression+C (SAG)

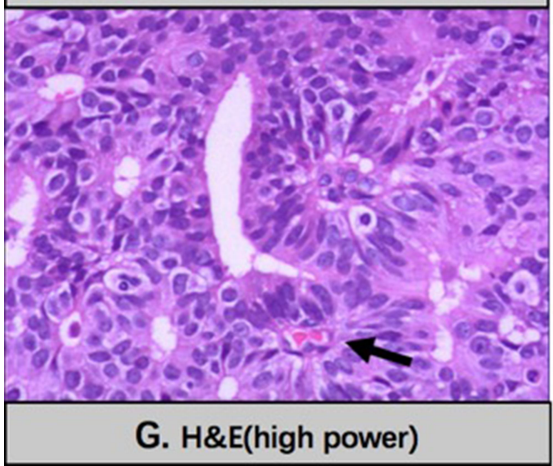

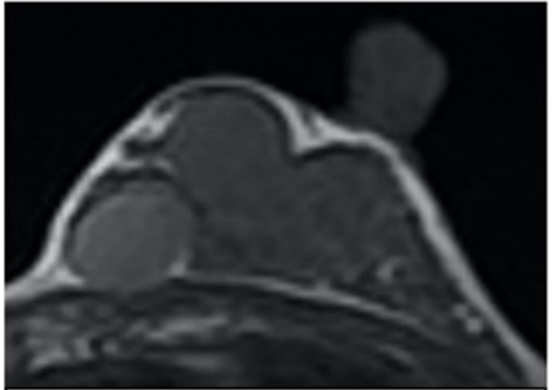

B. T1WI (TRA)

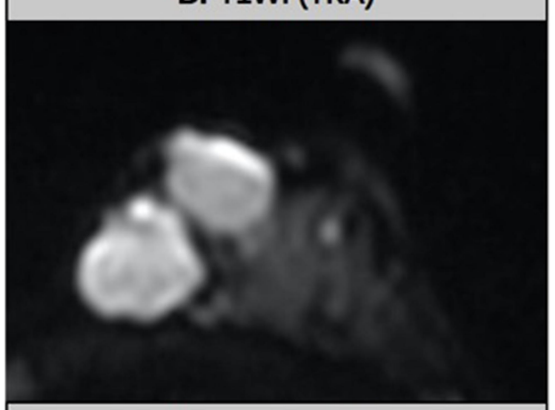

E. DWI $(b=800)$

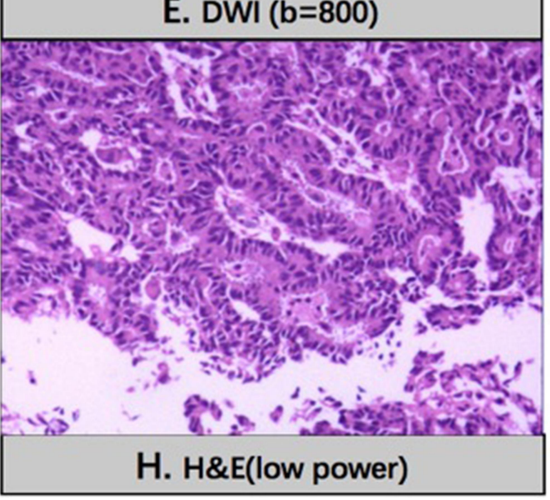

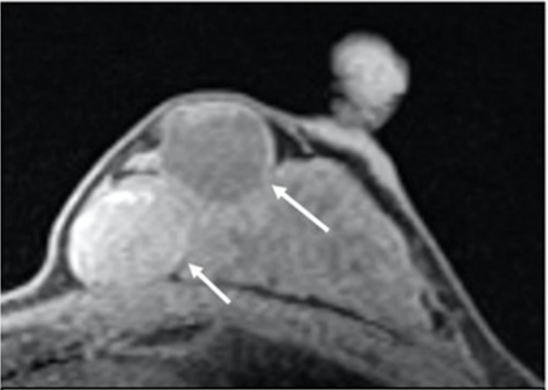

C. T1WI-fat suppression +C (TRA)

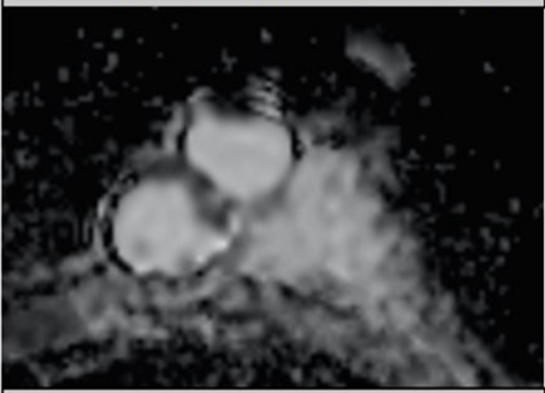

F. ADC

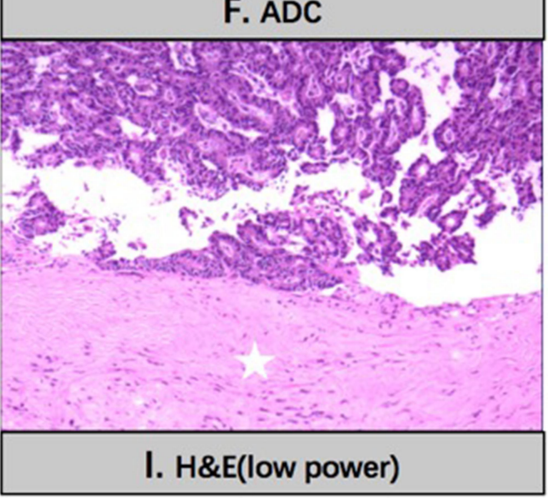

Figure 3 A 48-year-old woman with pure EPC in left breast. Axial T2WI with fat suppression (A) and TIWI (B) showed two mainly cystic masses, and one of the masses showed high intensity on TIWI images. Axial and sagittal fat-suppressed contrast-enhanced TIWI (C, D) showed the breast mass with circumscribed margin (white arrow). DWI (E) and ADC map (F) showed high signal mass. (G), H\&E stain (high power) showed the fibrovascular axis (black arrow), and papillary hyperplasia was seen at low power (H). (I), H\&E stain (low power) showed the tumor cells did not invade the fibrous capsule (pentagram).

of a high EPC risk stratification. The capsule wall of EPCs corresponds to a thick fibrous capsule on pathology. Comparative analysis with pathology revealed that lesions with not circumscribed of margins showed clusters of irregular tumor cells infiltrating the adjacent stroma. There was no significant difference in tumor size between the EPCs and EPCs with invasion, but we observed that the larger tumors increased the risk. We found that nearly half of the EPC with invasion group showed hyperintensity on T1WI, while the probability was low in the EPC group. Pathology confirmed that these lesions contained mucus or blood degradative components in cyst fluid. We inferred that the hemorrhage may be related to the rich blood supply and tumor fragility. The mucous composition may be due to the presence of a dilated catheter in the cystic space, which the tumor stimulates to secretion. However, whether this means high aggressiveness remains to be further examined. Another discovery was that more than half of the EPC with invasion group showed nonmass enhancement surrounding. These cases with nonmass enhancement surrounding were all confirmed to be associated with ductal carcinoma in situ (DCIS). According to past research, ${ }^{16}$ EPC was also thought to be classified into three main subtypes: EPC alone, EPC with surrounding DCIS, and EPC associated with invasive carcinoma. The imaging feature of non-mass enhancement surroundings can help to determine the presence or absence of DCIS and, to some extent, may be indicative of higher risk stratification. The dilated duct surrounding, we speculated, might be caused by the tumor obstructing 
Table 3 Treatment in Different EPC Types

\begin{tabular}{|c|c|c|}
\hline & $\begin{array}{l}\text { EPC } \\
(n=19)\end{array}$ & $\begin{array}{l}\text { EPC with Invasion } \\
(n=I I)\end{array}$ \\
\hline \multicolumn{3}{|l|}{ Types of operation } \\
\hline Breast-conserving surgery & 14 (46.7\%) & $4(13.3 \%)$ \\
\hline Simple mastectomy & $5(16.7 \%)$ & $7(23.3 \%)$ \\
\hline \multicolumn{3}{|l|}{ Sentinel lymph node } \\
\hline Positive & $0(0.0 \%)$ & I (3.3\%) \\
\hline Negative & $19(63.3 \%)$ & $10(33.3 \%)$ \\
\hline \multicolumn{3}{|l|}{ Axillary lymph node } \\
\hline Positive & $0(0.0 \%)$ & I (3.3\%) \\
\hline Negative & $19(63.3 \%)$ & $10(33.3 \%)$ \\
\hline $\begin{array}{l}\text { Puncture pathology } \\
\text { coincidence rate } \\
\text { Core needle biopsy (CNB) }\end{array}$ & & \\
\hline Concordant & $5(22.7 \%)$ & $3(13.6 \%)$ \\
\hline Not concordant & $9(40.9 \%)$ & $5(22.7 \%)$ \\
\hline \multicolumn{3}{|l|}{$\begin{array}{l}\text { Vacuum-assisted biopsy } \\
\text { (VAB) }\end{array}$} \\
\hline Concordant & $3(37.5 \%)$ & $2(25.0 \%)$ \\
\hline Not concordant & $2(25.0 \%)$ & I (I2.5\%) \\
\hline
\end{tabular}

the mammary duct. The dilated duct surrounding may not be helpful in determining EPC risk stratification, but they may help us to infer the mammary ductal origin lesions. We also tried to study the relationship between the proportion of solid components in the tumors and the pathological classification. We found that mainly cystic mass appeared only in EPC cases, and mainly cystic mass tended to be the low-risk stratification, but there was no significant difference. We need to expand the sample size to determine if this is an accident. Both the ADC value, ADC ratio and TIC pattern play a limited role in determining the risk stratification of EPCs. In this study, one case of axillary lymph node enlargement was found in patients with EPC with invasion and was pathologically confirmed as lymph node metastasis, and lymph node enlargement was not found in pure EPC. Usually, EPC without invasion has a very favorable prognosis, and few cases have lymph node metastases reported. ${ }^{17}$ Our study supports this statement, and the presence of axillary lymph node metastasis, although infrequent, may indicate a higher risk stratification.

In this study, the pathological concordance rate was low, especially using CNB. We analyzed that the reason might be that there was too little biopsy tissue to make a clear diagnosis. Only papillary lesions can be identified in most of the pathologic findings. Moreover, the presence of a breakthrough fibrous capsule cannot be determined. Remarkably, pathological examination revealed that the infiltrating part of the tumor stroma often occurred in the solid component of the cyst wall in EPCs with invasion.

Table 4 The Risk Stratification for EPC of the Breast and Information of Pathology

\begin{tabular}{|c|c|c|c|c|}
\hline & $\operatorname{EPC}(n=\mid 9)$ & EPC with Invasion $(n=I I)$ & Statistical value & $P$ value \\
\hline ER & & & -1.89 & 0.06 \\
\hline Positive & 19 & 9 & & \\
\hline Negative & 0 & 2 & & \\
\hline PR & & & -1.31 & 0.19 \\
\hline Positive & 19 & 10 & & \\
\hline Negative & 0 & I & & \\
\hline Her-2 & & & -1.31 & 0.19 \\
\hline Positive & 0 & 1 & & \\
\hline Negative & 19 & 10 & & \\
\hline $\mathrm{Ki}-67$ & & & -2.11 & 0.04 \\
\hline$<20 \%$ & 9 & 1 & & \\
\hline$\geq 20 \%$ & 10 & 10 & & \\
\hline Molecular subtype & & & -2.30 & 0.02 \\
\hline Luminal A & 9 & I & & \\
\hline Luminal B & 10 & 9 & & \\
\hline Her-2 positive & 0 & I & & \\
\hline TNBC & 0 & 0 & & \\
\hline
\end{tabular}

Abbreviations: ER, estrogen receptor; PR, progesterone receptor; Her-2, human epidermal growth factor receptor 2; TNBC, triple-negative breast cancer. 
Therefore, for preoperative evaluations of the lesion, we propose that MRI-guided puncture should be performed to improve the puncture protocol and the positive diagnosis rate of biopsy.

We further analyzed the pathological information of EPCs and EPCs with invasion, and found that higher Ki-67 levels were more common in patients with EPCs with invasion. Ki67 is correlated with a high mitotic count and can be used as a marker for evaluating the degree of cellularity. This indicates a higher level of tumor cell proliferation in the EPC with invasion group, and further demonstrates higher risk stratification in EPC with invasion. Molecular classification is an important prognostic indicator of breast cancer. According to the literature, most patients with EPCs exhibit an ER-positive, PR-positive phenotype. ${ }^{14,18,19}$ In our study, all EPC cases were of the luminal phenotype, which also suggested a good prognosis; however, in EPC with invasion group, one case was the Her-2-positive phenotype, which suggested a relatively poor prognosis. According to the Ki-67 level, ER/PR status, and HER2 expression, EPCs with invasion showed relatively highrisk stratification.

The application of the above MRI phenotypes as well as $\mathrm{Ki}-67$ levels to risk stratification analysis is of great significance for guiding clinical treatment. Since axillary lymph node metastasis is rare for pure EPCs, the precise axillary staging would not be recommended for every EPC patients and should be determined according to both context- and patient-specific, however, at present has not been clearly established for EPC patients. ${ }^{20,21}$ For EPC patients with low-risk stratification indicated above and negative axillary lymph node examination, we suggested follow-up observation. For EPC patients with higher risk stratification indicated above and accompanied by positive axillary lymph nodes, sentinel lymph node biopsy (SLNB) is feasible for axillary staging and further treatment.

There are some limitations in our study. First, as this study was a multicenter study, the MR model was not unified, and neither was the scanning sequence for the breast; however, these factors do not affect our diagnosis. Moreover, we obtained an abundant number of cases for a retrospective review and a more comprehensive understanding of MRI performance. Second, the proposed MRguided puncture procedure is only an assumption of ours, and further research plans are needed.

\section{Conclusion}

EPC of the breast is a rare tumor. This study showed that margin and age of patients as well as Ki-67 levels could be used to assess the EPC risk stratification, which could be conveniently introduced in clinical practice for guiding treatment and predicting prognosis. In addition, our study indicated that a larger tumor, non-mass enhancement surrounding and axillary lymph node metastasis may be suggestive of high-risk stratification. In conclusion, MRI phenotypes may provide additional information for the risk stratification of EPCs.

\section{Author Contributions}

Yuan Guo and Wen-Jie Tang conceived and designed the study and wrote the manuscript. All authors contributed to data analysis, drafting and reviewing of the article; gave final approval of the version to be published; have agreed on the journal to which the article has been submitted; and agree to be accountable for all aspects of the work.

\section{Funding}

This study was supported by the National Natural Science Foundation of China, No. 81901711.

\section{Disclosure}

The authors report no conflicts of interest in this work.

\section{References}

1. Fayanju OM, Ritter J, Gillanders WE, et al. Therapeutic management of intracystic papillary carcinoma of the breast: the roles of radiation and endocrine therapy. Am J Surg. 2007;194(4):497-500. doi:10.1016/j.amjsurg.2007.06.016

2. Mogal H, Brown DR, Isom S, Griffith K, Howard-McNatt M. Intracystic papillary carcinoma of the breast: a SEER database analysis of implications for therapy. Breast. 2016;27:87-92. doi:10.1016/ j.breast.2016.01.003

3. Tan PH, Schnitt SJ, van de Vijver MJ, Ellis IO, Lakhani SR. Papillary and neuroendocrine breast lesions: the WHO stance. Histopathology. 2015;66(6):761-770. doi:10.1111/his.12463

4. board Wcote. Breast Tumours. 5th ed. Lyon: IARC Press; 2019.

5. Li X, Xu Y, Ye H, Qin S, Hou F, Liu W. Encapsulated papillary carcinoma of the breast: a clinicopathological study of 49 cases. Curr Probl Cancer. 2018;42(3):291-301. doi:10.1016/j.currproblcancer.2018.03.005

6. George K, Anna Z, Evanthia K, Vassilios K. Encapsulated papillary carcinoma of the breast: an overview. $J$ Cancer Res Ther. 2013;9:564-570. doi:10.4103/0973-1482.126448

7. Collins LC, Schnitt SJ. Papillary lesions of the breast: selected diagnostic and management issues. Histopathology. 2008;52:20-29. doi:10.1111/j.1365-2559.2007.02898.x

8. Calderaro J, Espie M, Duclos J, et al. Breast intracystic papillary carcinoma: an update. Breast J. 2009;15(6):639-644. doi:10.1111/ j.1524-4741.2009.00823.x

9. Akladios CY, Roedlich MN, Bretz-Grenier MF, Croce S, Mathelin C. Intracystic papillary carcinoma of the breast: a diagnostic challenge with major clinical impact. Anticancer Res. 2014;34:5017-5020.

10. Eiada R, Chong J, Kulkarni S, Goldberg F, Muradali D. Papillary lesions of the breast: MRI, ultrasound, and mammographic appearances. AJR Am J Roentgenol. 2012;198(2):264-271. doi:10.2214/AJR.11.7922 
11. Sarica O, Uluc F, Tasmali D. Magnetic resonance imaging features of papillary breast lesions. Eur $J$ Radiol. 2014;83(3):524-530. doi:10.1016/j.ejrad.2013.12.007

12. Regan JP, Casaubon JT, Genelus-Dominique E. Synchronous invasive ductal carcinoma in encapsulated papillary ductal carcinoma. J Surg Case Rep. 2016;2016:rjw141. doi:10.1093/jscr/rjw141

13. Yilmaz R, Comert RG, Aliyev S, et al. Encapsulated papillary carcinoma in a man with gynecomastia: ultrasonography, mammography and magnetic resonance imaging features with pathologic correlation. Eur J Breast Health. 2018;14:127-131.

14. Jiang T, Tang W, Gu Y, Xu M, Yang W, Peng W. Magnetic resonance imaging features of breast encapsulated papillary carcinoma. J Comput Assist Tomogr. 2018;42:536-541. doi:10.1097/RCT. 0000000000000737

15. Morris C, Lee C. ACR BI-RADS ${ }^{\circledR}$ Magnetic Resonance Imaging ACR BI-RADS ${ }^{\circledR}$ Atlas, Breast Imaging Reporting and Data System. Reston, VA: American College of Radiology; 2013.

16. Baykara M, Coskun U, Demirci U, et al. Intracystic papillary carcinoma of the breast: one of the youngest patient in the literature. Med Oncol. 2010;27(4):1427-1428. doi:10.1007/s12032-009-9290-0
17. Mulligan AM, O'Malley FP. Metastatic potential of encapsulated (intracystic) papillary carcinoma of the breast: a report of 2 cases with axillary lymph node micrometastases. Int J Surg Pathol. 2007;15:143-147. doi:10.1177/1066896906299119

18. Wynveen CA, Nehhozina T, Akram M, et al. Intracystic papillary carcinoma of the breast: an in situ or invasive tumor? Results of immunohistochemical analysis and clinical follow-up. Am J Surg Pathol. 2011;35:1-14. doi:10.1097/PAS.0b013e3181fbe20a

19. Rakha EA, Gandhi N, Climent F, et al. Encapsulated papillary carcinoma of the breast: an invasive tumor with excellent prognosis. Am J Surg Pathol. 2011;35:1093-1103. doi:10.1097/PAS.0b013e31821b3f65

20. Steponaviciene L, Gudaviciene D, Briediene R, Petroska D, Garnelyte A. Diagnosis, treatment, and outcomes of encapsulated papillary carcinoma: a single institution experience. Acta Med Litu. 2018;25:66-75.

21. Hassan Z, Boulos F, Abbas J, El Charif M, Assi H, Sbaity E. Intracystic papillary carcinoma: clinical presentation, patterns of practice, and oncological outcomes. Breast Cancer Res Treat. 2020;182(2):317-323. doi:10.1007/s10549-020-05680-9

\section{Publish your work in this journal}

Cancer Management and Research is an international, peer-reviewed open access journal focusing on cancer research and the optimal use of preventative and integrated treatment interventions to achieve improved outcomes, enhanced survival and quality of life for the cancer patient.
The manuscript management system is completely online and includes a very quick and fair peer-review system, which is all easy to use. Visit http://www.dovepress.com/testimonials.php to read real quotes from published authors. 